\title{
Tracking the Rotor Time Constant of an Induction Motor Traction Drive for HEVs
}

\author{
Kaiyu Wang, John Chiasson, Marc Bodson and Leon M. Tolbert
}

\begin{abstract}
Field-oriented control methodology requires knowledge of the machine parameters, and in particular the rotor time constant. The interest here is in tracking the value of $T_{R}$ as it changes due to Ohmic heating so that an accurate value is available to estimate the rotor flux for a field oriented controller. The approach presented here is a nonlinear least-squares method that uses only measurements of the stator currents and voltages along with the rotor speed. The nature of this technique lends itself directly to an online implementation and therefore can be used to track the rotor time constant. Experimental results are presented to demonstrate the validity of the approach.
\end{abstract}

Keywords - Induction Motor, Rotor Time Constant, Parameter Identification

\section{INTRODUCTION}

The field-oriented control method provides a means to obtain high performance control of an induction machine for use in applications such as traction drives. This fieldoriented control methodology requires knowledge of the machine parameters, and in particular the rotor time constant which can vary due to Ohmic heating. The problem is further complicated by the fact that rotor variables are not usually available for measurement. The induction motor parameters are $M$ (the mutual inductance), $L_{S}, L_{R}$ (the stator and rotor inductances), $R_{S}, R_{R}$ (the stator and rotor resistances), $J$ (the inertia of the rotor) which are required for field oriented control. Standard methods for the estimation of induction motor parameters include the locked rotor test, the no-load test, and the standstill frequency response test. However, these approaches cannot be used online, that is, during normal operation of the machine. For example, field oriented control requires knowledge of the rotor time constant $T_{R}=L_{R} / R_{R}$ (which varies significantly due to Ohmic heating) in order to estimate the rotor flux linkages. The interest here is in tracking the value of $T_{R}$ as it changes. The approach is a nonlinear least-squares method using measurements of the stator currents and voltages along with the rotor speed. Due to the nature of this technique, it lends itself directly to an online implementation and therefore can be used to track the rotor time constant.

K. Wang, J. Chiasson and L. Tolbert are with the ECE Department, University of Tennessee, Knoxville, TN 37996. wkaiyu@utk.edu, chiasson@utk.edu, tolbert@utk.edu.

M. Bodson is with the ECE Department, University of Utah, Salt Lake City, UT 84112 bodson@ece.utah.edu

L. M. Tolbert is also with Oak Ridge National Laboratory, Oak Ridge TN. tolbertlm@ornl.gov

Drs. Chiasson and Tolbert would like to thank Oak Ridge National Laboratory for partially supporting this work through the UT/Battelle contract no. 4000007596. Dr. Tolbert would also like to thank the National Science Foundation for partially supporting this work through contract NSF ECS-0093884.
It turns out that, as the rotor state variables are not available measurements, the system identification model cannot be made linear in the parameters without overparameterizing the model. In the work here, the model is reformulated so that it is a nonlinear system identification problem that is not overparameterized. Further, it is shown how to actually solve for parameter vector that minimizes the residual error. This proposed method improves upon the linear least-squares approach formulated in [1][2]. The work in [1][2] was limited in that the acceleration was required to be small and that the iterative method used to solve the least squares problem was not guaranteed to converge nor necessarily achieve the minimum. Here, elimination theory [3][4] is used to solve the nonlinear least squares problem which in turn guarantees the minimum is found without any requirements on the machine's speed or acceleration when collecting the data; the data need only be sufficiently rich as described in the paper. Experimental results are presented to demonstrate the validity of the approach.

A combined parameter identification and velocity estimation problem is discussed in [5][6][7]. Here the velocity estimation problem is not considered, but the velocity is allowed to vary. For a summary of the various techniques for tracking the rotor time constant, the reader is referred to the recent survey [8], the recent paper [9] and to the book [10].

The paper is organized as follows. Section II introduces a standard induction motor model expressed in the rotor coordinates. Then, an overparameterized model which is linear in the unknown parameters is derived and discussed. Section IV presents the identification scheme for the rotor time constant by reducing the overparameterized linear model to a nonlinear model which is not overparameterized. An approach to solve the resulting nonlinear least-squares identification problem is presented and shown to guarantee the minimum least-squares solution is found. Section $\mathrm{V}$ presents the results of the identification algorithm with both simulated and experimental data.

\section{Induction Motor Model}

Standard models of induction machines are available in the literature. Parasitic effects such as hysteresis, eddy currents, magnetic saturation, and others are generally neglected. Consider the state space model of the system given 
by (cf. [11][12])

$$
\begin{aligned}
\frac{d \omega}{d t} & =\frac{M n_{p}}{J L_{R}}\left(i_{S b} \psi_{R a}-i_{S a} \psi_{R b}\right)-\frac{\tau_{L}}{J} \\
\frac{d \psi_{R a}}{d t} & =-\frac{1}{T_{R}} \psi_{R a}-n_{p} \omega \psi_{R b}+\frac{M}{T_{R}} i_{S a} \\
\frac{d \psi_{R b}}{d t} & =-\frac{1}{T_{R}} \psi_{R b}+n_{p} \omega \psi_{R a}+\frac{M}{T_{R}} i_{S b} \\
\frac{d i_{S a}}{d t} & =\frac{\beta}{T_{R}} \psi_{R a}+\beta n_{p} \omega \psi_{R b}-\gamma i_{S a}+\frac{1}{\sigma L_{S}} u_{S a} \\
\frac{d i_{S b}}{d t} & =\frac{\beta}{T_{R}} \psi_{R b}-\beta n_{p} \omega \psi_{R a}-\gamma i_{S b}+\frac{1}{\sigma L_{S}} u_{S b}
\end{aligned}
$$

where $\omega=d \theta / d t$ with $\theta$ the position of the rotor, $n_{p}$ is the number of pole pairs, and $i_{S a}, i_{S b}$ are the (two phase equivalent) stator currents and $\psi_{R a}, \psi_{R b}$ are the (two phase equivalent) rotor fluxes.

The parameters of the model are the five electrical parameters, $R_{S}$ and $R_{R}$ (the stator and rotor resistances), $M$ (the mutual inductance), $L_{S}$ and $L_{R}$ (the stator and rotor inductances), and the two mechanical parameters, $J$ (the inertia of the rotor) and $\tau_{L}$ (the load torque). The symbols

$$
\begin{array}{ll}
T_{R}=L_{R} / R_{R} & \sigma=1-M^{2} /\left(L_{S} L_{R}\right) \\
\beta=M /\left(\sigma L_{S} L_{R}\right) & \gamma=R_{S} /\left(\sigma L_{S}\right)+M^{2} R_{R} /\left(\sigma L_{S} L_{R}^{2}\right)
\end{array}
$$

have been used to simplify the expressions. $T_{R}$ is referred to as the rotor time constant while $\sigma$ is called the total leakage factor.

This model is transformed into a coordinate system attached to the rotor. For example, the current variables are transformed according to

$$
\left[\begin{array}{l}
i_{S x} \\
i_{S y}
\end{array}\right]=\left[\begin{array}{rr}
\cos \left(n_{p} \theta\right) & \sin \left(n_{p} \theta\right) \\
-\sin \left(n_{p} \theta\right) & \cos \left(n_{p} \theta\right)
\end{array}\right]\left[\begin{array}{c}
i_{S a} \\
i_{S b}
\end{array}\right] .
$$

The transformation simply projects the vectors in the $(a, b)$ frame onto the axes of the moving coordinate frame. An advantage of this transformation is that the signals in the moving frame (i.e., the $(x, y)$ frame) typically vary slower than those in the $(a, b)$ frame (they vary at the slip frequency rather than at the stator frequency). At the same time, the transformation does not depend on any unknown parameter in contrast to the field-oriented $d / q$ transformation. The stator voltages and the rotor fluxes are transformed as the currents resulting in the following model ([2])

$$
\begin{aligned}
\frac{d i_{S x}}{d t} & =\frac{u_{S x}}{\sigma L_{S}}-\gamma i_{S x}+\frac{\beta}{T_{R}} \psi_{R x}+n_{p} \beta \omega \psi_{R y}+n_{p} \omega i_{S y}(3) \\
\frac{d i_{S y}}{d t} & =\frac{u_{S y}}{\sigma L_{S}}-\gamma i_{S y}+\frac{\beta}{T_{R}} \psi_{R y}-n_{p} \beta \omega \psi_{R x}-n_{p} \omega i_{S x}(4) \\
\frac{d \psi_{R x}}{d t} & =\frac{M}{T_{R}} i_{S x}-\frac{1}{T_{R}} \psi_{R x} \\
\frac{d \psi_{R y}}{d t} & =\frac{M}{T_{R}} i_{S y}-\frac{1}{T_{R}} \psi_{R y} \\
\frac{d \omega}{d t} & =\frac{M n_{p}}{J L_{R}}\left(i_{S y} \psi_{R x}-i_{S x} \psi_{R y}\right)-\frac{\tau_{L}}{J} .
\end{aligned}
$$

\section{LineAR Overparameterized MODEL}

As stated in the introduction, the interest here is in tracking the value of $T_{R}$ as it changes due to Ohmic heating so that an accurate value is available to estimate the flux for a field oriented controller. However, the stator resistance value $R_{S}$ will also vary due to Ohmic heating so that it must also be taken into account. The electrical parameters $M, L_{S}, \sigma$ are assumed to be known and not varying. Measurements of the stator currents $i_{S a}, i_{S b}$ and voltages $u_{S a}, u_{S b}$ as well as the position $\theta$ of the rotor are assumed to be available; velocity is then reconstructed from the position measurements. However, the rotor flux linkages are not assumed to be measured.

Standard methods for parameter estimation are based on equalities where known signals depend linearly on unknown parameters. However, the induction motor model described above does not fit in this category unless the rotor flux linkages are measured. The first step is to eliminate the fluxes $\psi_{R x}, \psi_{R y}$ and their derivatives $d \psi_{R x} / d t, d \psi_{R y} / d t$. The four equations (3), (4), (5), (6) can be used to solve for $\psi_{R x}, \psi_{R y}, d \psi_{R x} / d t, d \psi_{R y} / d t$, but one is left without another independent equation to set up a regressor system for the identification algorithm. A new set of independent equations are found by differentiating equations (3) and (4) to obtain

$$
\begin{aligned}
\frac{1}{\sigma L_{s}} \frac{d u_{S x}}{d t}= & \frac{d^{2} i_{S x}}{d t^{2}}+\gamma \frac{d i_{S x}}{d t}-\frac{\beta}{T_{R}} \frac{d \psi_{R x}}{d t}-n_{p} \beta \omega \frac{d \psi_{R y}}{d t} \\
& -n_{p} \beta \psi_{R y} \frac{d \omega}{d t}-n_{p} \omega \frac{d i_{S y}}{d t}-n_{p} i_{S y} \frac{d \omega}{d t}
\end{aligned}
$$

and

$$
\begin{aligned}
\frac{1}{\sigma L_{s}} \frac{d u_{S y}}{d t}= & \frac{d^{2} i_{S y}}{d t^{2}}+\gamma \frac{d i_{S y}}{d t}-\frac{\beta}{T_{R}} \frac{d \psi_{R y}}{d t}+n_{p} \beta \omega \frac{d \psi_{R x}}{d t} \\
& +n_{p} \beta \psi_{R x} \frac{d \omega}{d t}+n_{p} \omega \frac{d i_{S x}}{d t}+n_{p} i_{S x} \frac{d \omega}{d t} .
\end{aligned}
$$

Next, equations (3), (4), (5), (6) are solved for $\psi_{R x}, \psi_{R y}$, $d \psi_{R x} / d t, d \psi_{R y} / d t$ and substituted into equations (8) and (9) to obtain

$$
\begin{aligned}
0 & =-\frac{d^{2} i_{S x}}{d t^{2}}+\frac{d i_{S y}}{d t} n_{p} \omega+\frac{1}{\sigma L_{S}} \frac{d u_{S x}}{d t}-\left(\gamma+\frac{1}{T_{R}}\right) \frac{d i_{S x}}{d t} \\
& -i_{S x}\left(-\frac{\beta M}{T_{R}^{2}}+\frac{\gamma}{T_{R}}\right)+i_{S y} n_{p} \omega\left(\frac{1}{T_{R}}+\frac{\beta M}{T_{R}}\right)+\frac{u_{S x}}{\sigma L_{S} T_{R}} \\
& +n_{p} \frac{d \omega}{d t} i_{S y}-n_{p} \frac{d \omega}{d t} \frac{1}{\sigma L_{S}\left(1+n_{p}^{2} \omega^{2} T_{R}^{2}\right)} \times \\
& \left(-\sigma L_{S} T_{R} \frac{d i_{S y}}{d t}-\gamma i_{S y} \sigma L_{S} T_{R}-i_{S x} n_{p} \omega \sigma L_{S} T_{R}\right. \\
& -\frac{d i_{S x}}{d t} n_{p} \omega \sigma L_{S} T_{R}^{2}-\gamma i_{S x} n_{p} \omega \sigma L_{S} T_{R}^{2}+i_{S y} n_{p}^{2} \omega^{2} \sigma L_{S} T_{R}^{2} \\
& \left.+n_{p} \omega T_{R}^{2} u_{S x}+T_{R} u_{S y}\right)
\end{aligned}
$$




$$
\begin{aligned}
0 & =-\frac{d^{2} i_{S y}}{d t^{2}}-\frac{d i_{S x}}{d t} n_{p} \omega+\frac{1}{\sigma L_{S}} \frac{d u_{S y}}{d t}-\left(\gamma+\frac{1}{T_{R}}\right) \frac{d i_{S y}}{d t} \\
& -i_{S y}\left(-\frac{\beta M}{T_{R}^{2}}+\frac{\gamma}{T_{R}}\right)-i_{S x} n_{p} \omega\left(\frac{1}{T_{R}}+\frac{\beta M}{T_{R}}\right)+\frac{u_{S y}}{\sigma L_{S} T_{R}} \\
& -n_{p} \frac{d \omega}{d t} i_{S x}+n_{p} \frac{d \omega}{d t} \frac{1}{\sigma L_{S}\left(1+n_{p}^{2} \omega^{2} T_{R}^{2}\right)} \times \\
& \left(-\sigma L_{S} T_{R} \frac{d i_{S x}}{d t}-\gamma i_{S x} \sigma L_{S} T_{R}+i_{S y} n_{p} \omega \sigma L_{S} T_{R}\right. \\
& +\frac{d i_{S y}}{d t} n_{p} \omega \sigma L_{S} T_{R}^{2}+\gamma i_{S y} n_{p} \omega \sigma L_{S} T_{R}^{2}+i_{S x} n_{p}^{2} \omega^{2} \sigma L_{S} T_{R}^{2} \\
& \left.-n_{p} \omega T_{R}^{2} u_{S y}+T_{R} u_{S x}\right) .
\end{aligned}
$$

This set of equations may be rewritten in regressor form as

$$
y(t)=W(t) K
$$

where $W \in \Re^{2 \times 8}, K \in \Re^{8}$ and $y \in \Re^{2}$ are given by

$$
\begin{aligned}
& W=\left[\begin{array}{cc}
-\frac{d i_{S x}}{d t} & -\frac{d i_{S x}}{d t}+n_{p} \omega i_{S y}+n_{p} \omega M \beta i_{S y}+\frac{u_{S x}}{\sigma L s} \\
-\frac{d i_{S y}}{d t} & -\frac{d i_{S y}}{d t}-n_{p} \omega i_{S x}-n_{p} \omega M \beta i_{S x}+\frac{u_{S y}}{\sigma L s}
\end{array}\right. \\
& M \beta i_{S x} \quad-i_{S x} \quad n_{p} \frac{d i_{S y}}{d t} \frac{d \omega}{d t}+n_{p}^{2}\left(\omega i_{S x} \frac{d \omega}{d t}-\omega^{2} \frac{d i_{S x}}{d t}\right) \\
& M \beta i_{S y} \quad-i_{S y} \quad-n_{p} \frac{d i_{S x}}{d t} \frac{d \omega}{d t}+n_{p}^{2}\left(\omega i_{S y} \frac{d \omega}{d t}-\omega^{2} \frac{d i_{S y}}{d t}\right) \\
& +n_{p}^{3} \omega^{3} i_{S y}(1+M \beta)+\frac{1}{\sigma L s}\left(n_{p}^{2} \omega^{2} u_{S x}-n_{p} u_{S y} \frac{d \omega}{d t}\right) \\
& -n_{p}^{3} \omega^{3} i_{S x}(1+M \beta)+\frac{1}{\sigma L s}\left(n_{p}^{2} \omega^{2} u_{S y}+n_{p} u_{S x} \frac{d \omega}{d t}\right) \\
& n_{p} i_{S y} \frac{d \omega}{d t}-n_{p}^{2} \omega^{2} i_{S x} \quad n_{p}^{2}\left(i_{s x} \omega \frac{d \omega}{d t}-\omega^{2} \frac{d i_{S x}}{d t}\right) \\
& -n_{p} i_{S x} \frac{d \omega}{d t}-n_{p}^{2} \omega^{2} i_{S y} \quad n_{p}^{2}\left(i_{s y} \omega \frac{d \omega}{d t}-\omega^{2} \frac{d i_{S y}}{d t}\right) \\
& n_{p}^{2}\left(\omega \frac{d i_{S x}}{d t} \frac{d \omega}{d t}-\omega^{2} \frac{d^{2} i_{S x}}{d t^{2}}\right)+\frac{d i_{S y}}{d t} n_{p}^{3} \omega^{3} \\
& n_{p}^{2}\left(\omega \frac{d i_{S y}}{d t} \frac{d \omega}{d t}-\omega^{2} \frac{d^{2} i_{S y}}{d t^{2}}\right)-\frac{d i_{S x}}{d t} n_{p}^{3} \omega^{3} \\
& \left.-\frac{n_{p}^{2}}{\sigma L_{2} s}\left(\omega u_{S x} \frac{d \omega}{d t}-\omega^{2} \frac{d u_{S x}}{d t}\right)\right] \text {, } \\
& \left.-\frac{n_{p}^{2}}{\sigma L s}\left(\omega u_{S y} \frac{d \omega}{d t}-\omega^{2} \frac{d u_{S y}}{d t}\right)\right] \\
& K \triangleq\left[\begin{array}{llllllll}
\gamma & \frac{1}{T_{R}} & \frac{1}{T_{R}^{2}} & \frac{\gamma}{T_{R}} & T_{R} & \gamma T_{R} & \gamma T_{R}^{2} & T_{R}^{2}
\end{array}\right]^{T}
\end{aligned}
$$

and

$$
\begin{aligned}
y \triangleq & {\left[\begin{array}{l}
\frac{d^{2} i_{S x}}{d t^{2}}-n_{p} i_{S y} \frac{d \omega}{d t}-n_{p} \omega \frac{d i_{S y}}{d t}-n_{p}^{2} \omega^{2} M \beta i_{S x} \\
\frac{d^{2} i_{S y}}{d t^{2}}+n_{p} i_{S x} \frac{d \omega}{d t}+n_{p} \omega \frac{d i_{S x}}{d t}-n_{p}^{2} \omega^{2} M \beta i_{S y}
\end{array}\right] } \\
& -\frac{d u_{S x} / d t}{\sigma L s} \\
& \left.-\frac{d v_{S y} / d t}{\sigma L s}\right] .
\end{aligned}
$$

As $\frac{M^{2}}{L_{R}}=(1-\sigma) L_{S}, M \beta=(1-\sigma) / \sigma, \gamma=\frac{R_{S}}{\sigma L_{S}}+$ $\frac{1}{\sigma L_{S}} \frac{1}{T_{R}} \frac{M^{2}}{L_{R}}=\frac{R_{S}}{\sigma L_{S}}+\frac{1}{\sigma L_{S}} \frac{1}{T_{R}}(1-\sigma) L_{S}$ it is seen that $y$ and $W$ depend only on known quantities while the unknowns $R_{S}, T_{R}$ are contained only within $K$.

Though the system regressor is linear in the parameters, one cannot use standard least-squares techniques as the system is overparameterized. Specifically,

$$
\begin{aligned}
& K_{3}=K_{2}^{2}, K_{4}=K_{1} K_{2}, K_{5}=1 / K_{2}, K_{6}=K_{1} / K_{2}, \\
& K_{7}=K_{1} / K_{2}^{2}, K_{8}=1 / K_{2}^{2}
\end{aligned}
$$

so that only the two parameters $K_{1}, K_{2}$ are independent. These two parameters determine $R_{S}$ and $T_{R}$ by

$$
\begin{aligned}
T_{R} & =1 / K_{2} \\
R_{S} & =\sigma L_{S} K_{1}-(1-\sigma) L_{S} K_{2} .
\end{aligned}
$$

\section{Least-Squares Identification [13][14][15]}

Equation (12) can be rewritten as

$$
y(n)=W(n) K
$$

where $n$ is the time instant at which a measurement is taken and $K$ is the vector of unknown parameters. If the constraint (13) is ignored, then the system is an overparameterized linear least-squares problem. In this case, theoretically an exact unique solution for the unknown parameter vector $K$ may be determined after several time instants. However, several factors contribute to errors which make equation (15) only approximately valid in practice. Specifically, both $y(n)$ and $W(n)$ are measured through signals that are noisy due to quantization and differentiation. Further, the dynamic model of the induction motor is only an approximate representation of the real system. These sources of error result in an inconsistent system of equations. To find a solution for such a system, the least-squares algorithm is used. Specifically, given $y(n)$ and $W(n)$ where $y(n)=W(n) K$, one defines

$$
E^{2}(K)=\sum_{n=1}^{N}|y(n)-W(n) K|^{2}
$$

as the residual error associated to a vector $K$. Then, the least-squares estimate $K^{*}$ is chosen such that $E^{2}(K)$ is minimized for $K=K^{*}$. The function $E^{2}(K)$ is quadratic and therefore has a unique minimum at the point where $\partial E^{2}(K) / \partial K=0$. Solving this expression for $K^{*}$ yields the least-squares solution to $y(n)=W(n) K$ as

$$
K^{*}=\left[\sum_{n=1}^{N} W^{T}(n) W(n)\right]^{-1}\left[\sum_{n=1}^{N} W^{T}(n) y(n)\right] .
$$

When the system model is overparameterized as in the application here, the expression (17) will lead to an ill conditioned solution for $K^{*}$. That is, small changes in the data $W(n), y(n)$ lead to large changes in the value computed for $K^{*}$. To get around this problem, a nonlinear least-squares approach is taken which involves minimizing

$$
E^{2}(K)=\sum_{n=1}^{N}|y(n)-W(n) K|^{2}=R_{y}-2 R_{W y}^{T} K+K^{T} R_{W} K
$$


subject to the constraints (13). On physical grounds, the parameters $K_{1}, K_{2}$ are constrained to

$$
0<K_{1}<\infty, 0<K_{2}<\infty .
$$

Also, based on physical grounds, the squared error $E^{2}(K)$ will be minimized in the interior of this region. Let

$$
\begin{aligned}
& E^{2}\left(K_{p}\right) \triangleq \sum_{n=1}^{N}|y(n)-W(n) K|_{\substack{K_{3}=K_{2}^{2} \\
K_{4}=K_{1} K_{2}}}^{2} \\
& =R_{y}-\left.2 R_{W y}^{T} K\right|_{\substack{K_{3}=K_{2}^{2} \\
K_{4}=K_{1} K_{2}}}+\left.\left(K^{T} R_{W} K\right)\right|_{\substack{K_{3}=K_{2}^{2} \\
K_{4}=K_{1} K_{2}}}
\end{aligned}
$$

where

$$
K_{p} \triangleq\left[\begin{array}{ll}
K_{1} & K_{2}
\end{array}\right]^{T} .
$$

As just explained, the minimum of (20) must occur in the interior of the region and therefore at an extremum point. This then entails solving the two equations

$$
\begin{aligned}
& r_{1}\left(K_{p}\right) \triangleq \frac{\partial E^{2}\left(K_{p}\right)}{\partial K_{1}}=0 \\
& r_{2}\left(K_{p}\right) \triangleq \frac{\partial E^{2}\left(K_{p}\right)}{\partial K_{2}}=0 .
\end{aligned}
$$

The partial derivatives in (21)-(22) are rational functions in the parameters $K_{1}, K_{2}$. Defining

$$
\begin{aligned}
& p_{1}\left(K_{p}\right) \triangleq K_{2}^{4} r_{1}\left(K_{p}\right)=K_{2}^{4} \frac{\partial E^{2}\left(K_{p}\right)}{\partial K_{1}} \\
& p_{2}\left(K_{p}\right) \triangleq K_{2}^{5} r_{2}\left(K_{p}\right)=K_{2}^{5} \frac{\partial E^{2}\left(K_{p}\right)}{\partial K_{2}}
\end{aligned}
$$

results in the $p_{i}\left(K_{p}\right)$ being polynomials in the parameters $K_{1}, K_{2}$ and having the same positive zero set (i.e., the same roots satisfying $\left.K_{i}>0\right)$ as the system (21)-(22). The degrees of the polynomials $p_{i}$ are given in the table below.

\begin{tabular}{|c|c|c|}
\hline & $\operatorname{deg} K_{1}$ & $\operatorname{deg} K_{2}$ \\
\hline$p_{1}\left(K_{p}\right)$ & 1 & 7 \\
\hline$p_{2}\left(K_{p}\right)$ & 2 & 8 \\
\hline
\end{tabular}

All possible solutions to this set may be found using elimination theory as is now summarized.

Solving Systems of Polynomial Equations [3][4]

The question at hand is "Given two polynomial equations $a\left(K_{1}, K_{2}\right)=0$ and $b\left(K_{1}, K_{2}\right)=0$, how does one solve them simultaneously to eliminate (say) $K_{2}$ ?". A systematic procedure to do this is known as elimination theory and uses the notion of resultants. Briefly, one considers $a\left(K_{1}, K_{2}\right)$ and $b\left(K_{1}, K_{2}\right)$ as polynomials in $K_{2}$ whose coefficients are polynomials in $K_{1}$. Then, for example, letting $a\left(K_{1}, K_{2}\right)$ and $b\left(K_{1}, K_{2}\right)$ have degrees 3 and 2 , respectively in $K_{2}$, they may be written in the form

$$
\begin{aligned}
& a\left(K_{1}, K_{2}\right)=a_{3}\left(K_{1}\right) K_{2}^{3}+a_{2}\left(K_{1}\right) K_{2}^{2}+a_{1}\left(K_{1}\right) K_{2}+a_{0}\left(K_{1}\right) \\
& b\left(K_{1}, K_{2}\right)=b_{2}\left(K_{1}\right) K_{2}^{2}+b_{1}\left(K_{1}\right) K_{2}+b_{0}\left(K_{1}\right) .
\end{aligned}
$$

The $n \times n$ Sylvester matrix, where $n=\operatorname{deg}_{K_{2}}\left\{a\left(K_{1}, K_{2}\right)\right\}+$ $\operatorname{deg}_{K_{2}}\left\{b\left(K_{1}, K_{2}\right)\right\}=3+2=5$, is defined by

$S_{a, b}\left(K_{1}\right)=\left[\begin{array}{ccccc}a_{0}\left(K_{1}\right) & 0 & b_{0}\left(K_{1}\right) & 0 & 0 \\ a_{1}\left(K_{1}\right) & a_{0}\left(K_{1}\right) & b_{1}\left(K_{1}\right) & b_{0}\left(K_{1}\right) & 0 \\ a_{2}\left(K_{1}\right) & a_{1}\left(K_{1}\right) & b_{2}\left(K_{1}\right) & b_{1}\left(K_{1}\right) & b_{0}\left(K_{1}\right) \\ a_{3}\left(K_{1}\right) & a_{2}\left(K_{1}\right) & 0 & b_{2}\left(K_{1}\right) & b_{1}\left(K_{1}\right) \\ 0 & a_{3}\left(K_{1}\right) & 0 & 0 & b_{2}\left(K_{1}\right)\end{array}\right]$.

The resultant polynomial is then defined by

$$
r\left(K_{1}\right)=\operatorname{Res}\left(a\left(K_{1}, K_{2}\right), b\left(K_{1}, K_{2}\right), K_{2}\right) \triangleq \operatorname{det} S_{a, b}\left(K_{1}\right)
$$

and is the result of eliminating the variable $K_{2}$ from $a\left(K_{1}, K_{2}\right)$ and $b\left(K_{1}, K_{2}\right)$. In fact, the following is true.

Theorem 1: Any solution $\left(K_{1}^{0}, K_{2}^{0}\right)$ of $a\left(K_{1}, K_{2}\right)=0$ and $b\left(K_{1}, K_{2}\right)=0$ must have $r\left(K_{1}^{0}\right)=0$. [3][4].

Though the converse of this theorem is not necessarily true, the finite number of solutions of $r\left(K_{1}\right)=0$ are the only possible candidates for the first coordinate (partial solutions) of the common zeros of $a\left(K_{1}, K_{2}\right)$ and $b\left(K_{1}, K_{2}\right)$. Whether or not such a partial solution results in a full solution is simply determined by back solving and checking the solution.

Using the polynomials (23)-(24), the variable $K_{1}$ is eliminated to obtain

$$
r\left(K_{1}\right) \triangleq \operatorname{Res}\left(p_{1}\left(K_{1}, K_{2}\right), p_{2}\left(K_{1}, K_{2}\right), K_{1}\right)
$$

where $\operatorname{deg}_{K_{1}}\left\{r\left(K_{1}\right)\right\}=20$. The parameter $K_{2}$ was chosen as the variable not eliminated because its degree is much higher than $K_{1}$ meaning it would have a larger (in dimension) Sylvester matrix. The positive roots of $r\left(K_{1}\right)=0$ are found which are then substituted into $p_{1}=0\left(\right.$ or $\left.p_{2}=0\right)$ to find the positive roots in $K_{2}$, etc. By this method of back solving, all possible (finite number) candidate solutions are found and one simply chooses the one that gives the smallest squared error.

Conditioning of the Nonlinear Least-Squares

\section{Problem}

After finding the solution that gives the minimal value for $E^{2}\left(K_{p}\right)$, one needs to know if the solution makes sense. For example, in the linear least-squares problem, there is a unique well defined solution provided that the regressor matrix $R_{W}$ is nonsingular (or in practical terms, its condition number is not too large). In the nonlinear case here, a Taylor series expansion about the computed minimum point $K_{p}^{*}=\left[K_{1}^{*}, K_{2}^{*}\right]^{T}$ gives $(i, j=1,2)$

$E^{2}\left(K_{p}\right)=E^{2}\left(K_{p}^{*}\right)+\frac{1}{2}\left[K_{p}-K_{p}^{*}\right]^{T} \frac{\partial^{2} E^{2}\left(K_{p}^{*}\right)}{\partial K_{i} \partial K_{j}}\left[K_{p}-K_{p}^{*}\right]+\cdots$

One then checks that the Hessian matrix $\frac{\partial^{2} E^{2}\left(K_{p}^{*}\right)}{\partial K_{i} \partial K_{j}}$ is positive definite as well as its condition number to ensure that the data is sufficiently rich to identify the parameters.

\section{Experimental Results}

A three phase, $230 \mathrm{~V}, 0.5 \mathrm{Hp}, 1735 \mathrm{rpm}\left(n_{p}=2\right.$ polepair) induction machine was used for the experiments. A 
4096 pulse/rev optical encoder was attached to the motor for position measurements. The motor was connected to a three-phase $60 \mathrm{~Hz}$ source through a switch. When the switch was closed, the stator currents and voltages along with the rotor position were sampled at $4 \mathrm{kHz}$. Filtered differentiation (using digital filters) was used for calculating the acceleration and the derivatives of the voltages and currents. Specifically, the signals were filtered with a lowpass digital Butterworth filter followed by reconstruction of the derivatives using $d x(t) / d t=(x(t)-x(t-T)) / T$ where $T$ is the sampling interval. The voltages and currents were put through a $3-2$ transformation to obtain the two phase equivalent voltages $u_{S a}, u_{S b}$ which are plotted in Figure 1. The sampled two phase equivalent cur-

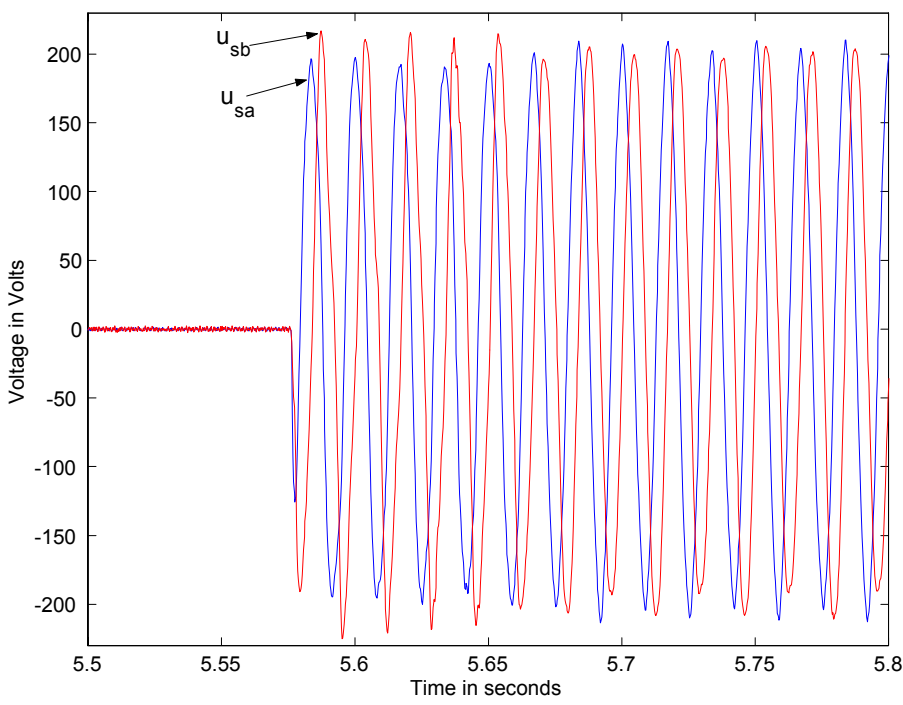

Fig. 1. Sampled two phase equivalent voltages $u_{S a}$ and $u_{S b}$.

rent $i_{S a}$ and it simulated response $i_{S a_{-} \text {sim }}$ are shown in Figure 2 (The simulated current will be discussed below). The phase $b$ current $i_{S b}$ is similar, but shifted by $\pi /\left(2 n_{p}\right)$. The calculated speed $\omega$ (from the position measurements) and the simulated speed $\omega_{\text {sim }}$ are shown in Figure 3 (the simulated speed $\omega_{\text {sim }}$ will be discussed below). Using the data $\left\{u_{S a}, u_{S b}, i_{S a}, i_{S b}, \theta\right\}$ collected between 5.57 sec to $5.8 \mathrm{sec}$, the quantities $u_{S x}, u_{S y}, d u_{S x} / d t, d u_{S y} / d t, i_{S x}, i_{S y}$ $d i_{S x} / d t, d i_{S y} / d t, d^{2} i_{S x} / d t^{2}, d^{2} i_{S y} / d t^{2}, \omega=d \theta / d t, d \omega / d t$ were calculated and the regressor matrices $R_{W}, R_{y}$ and $R_{W y}$ were computed. The procedure explained in Section IV was then carried out to compute $K_{1}, K_{2}$. In this case, there were three sets of extrema points that had positive values for all the $K_{i}$. The extremum value for $K_{1}, K_{2}$ that resulted in the minimum least-squares error was

$$
\begin{aligned}
& K 1=241.1024 \\
& K 2=7.5988
\end{aligned}
$$

Using (14), the motors electrical parameters are then

$$
\begin{aligned}
& T_{R}=0.1316 \mathrm{sec} \\
& R_{S}=5.0923 \Omega
\end{aligned}
$$

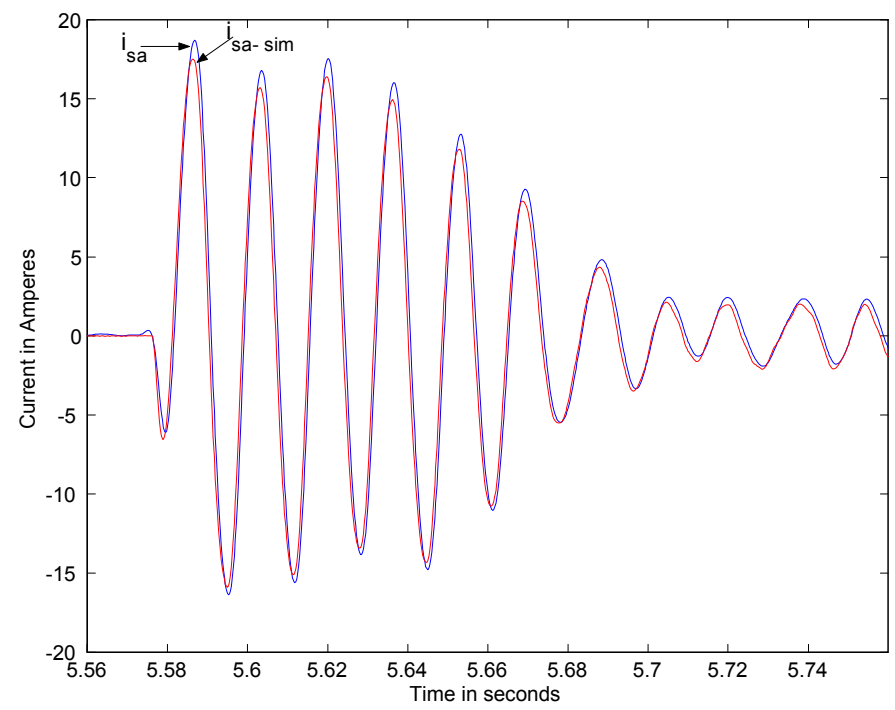

Fig. 2. Phase a current $i_{S a}$ and its simulated response $i_{S a \_s i m}$.

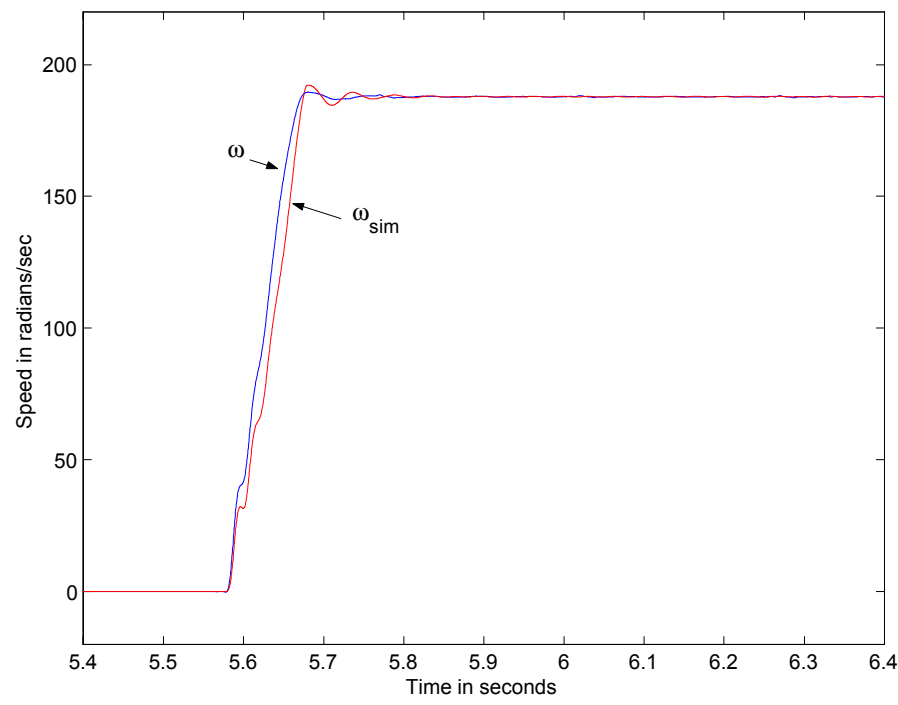

Fig. 3. Calculated speed $\omega$ and simulated speed $\omega_{\text {sim }}$.

By way of comparison, the stator resistance was measured using an Ohmmeter giving the value of 4.9 Ohms. The Hessian matrix was calculated at the minimum point according to (26) resulting in

$$
\left\{\frac{\partial^{2} E^{2}\left(K_{p}^{*}\right)}{\partial K_{i} \partial K_{j}}\right\}=\left[\begin{array}{cc}
1.9123 & 0.00412 \\
0.00412 & 570.0418
\end{array}\right]
$$

which is positive definite and has a condition number of $8.24 \times 10^{4}$.

\section{A. Simulation of the Experimental Motor}

Another useful way to evaluate the identified parameters (27) and (28) is to simulate the motor using these values and the measured voltages as input. The model (1) is now in terms of the parameters that can be estimated. The experimental voltages shown in Figure 1 were then used 
as input to a simulation of the model (1) using the parameter values from (27) and (28). The resulting phase $a$ current $i_{S a_{-} \text {sim }}$ from the simulation is shown in Figure 2 and corresponds well with the actual measured current $i_{S a}$. Similarly, the resulting speed $\omega_{\text {sim }}$ from the simulation is shown in Figure 3 where it is seen that the simulated speed is somewhat more oscillatory than the measured speed $\omega$.

\section{Conclusions}

In this paper, a method for estimating the rotor time constant and stator resistance of an induction machine was presented. The parameter model was formulated as a nonlinear least-squares problem and then solved using elimination theory. Experimental results showed a close correlation with simulations based on the identified parameters. An important advantage of the procedure is that it can be used online, i.e., during regular operation of the machine, its parameter values can be continuously updated assuming sufficient excitation of the machine. This method has been extended by the authors to the case of estimating all of the identifiable parameters of the induction motor [16]

\section{REFERENCES}

[1] J. Stephan, M. Bodson, and J. Chiasson, "Real-time estimation of induction motor parameters", IEEE Transactions on Industry Applications, vol. 30, no. 3, pp. 746-759, May/June 1994.

[2] J. Stephan, "Real-time estimation of the parameters and fluxes of induction motors", Master's thesis, Carnegie Mellon University, 1992.

[3] David Cox, John Little, and Donal O'Shea, IDEALS, VARIETIES, AND ALGORITHMS An Introduction to Computational Algebraic Geometry and Commutative Algebra, Second Edition, Springer-Verlag, 1996.

[4] Joachim von zur Gathen and Jürgen Gerhard, Modern Computer Algebra, Cambridge University Press, 1999.

[5] M. Vélez-Reyes, K. Minami, and G. Verghese, "Recursive speed and parameter estimation for induction machines", in Proceedings of the IEEE Industry Applications Conference, 1989, San Diego, California.

[6] Miguel Vélez-Reyes, W. L. Fung, and J. E. Ramos-Torres, "Developing robust algorithms for speed and parameter estimation in induction machines", in Proceedings of the IEEE Conference on Decision and Control, 2001, Orlando FL.

[7] Miguel Vélez-Reyes and George Verghese, "Decomposed algorithms for speed and parameter estimation in induction machines", in Proceedings of the IFAC Nonlinear Control Systems Design Symposium, 1992, pp. 156-161, Bordeaux, France.

[8] Hamid A. Toliyat, Emil Levi, and Mona Raina, "A review of RFO induction motor parameter estimation techniques", IEEE Transactions on Energy Conversion, vol. 18, no. 2, pp. 271-283, June 2003.

[9] Miguel Vélez-Reyes, Milan Mijalkovic, Aleksandar M. Stankovic, Silva Hiti, and James Nagashima, "Output selection for tuning of field oriented controllers: Steady state analysis", in Conference Record of Industry Applications Society, October 2003, Salt Lake City UT.

[10] Peter Vas, Parameter estimation, condition monitoring, and diagnosis of electrical machines, Oxford: Clarendon Press, 1993.

[11] R. Marino, S. Peresada, and P. Valigi, "Adaptive input-output linearizing control of induction motors", IEEE Transactions on Automatic Control, vol. 38, no. 2, pp. 208-221, February 1993.

[12] M. Bodson, J. Chiasson, and R. Novotnak, "High performance induction motor control via input-output linearization", IEEE Control Systems Magazine, vol. 14, no. 4, pp. 25-33, August 1994.

[13] Lennart Ljung, System Identification: Theory for the User, Prentice-Hall Inc., 1986.

[14] T. Söderström and P. Stoica, System Identification, PrenticeHall International, 1989.
[15] Shankar Sastry and Marc Bodson, Adaptive Control: Stability, Convergence, and Robustness, Prentice Hall, 1989.

[16] Kaiyu Wang, John Chiasson, Marc Bodson, and Leon Tolbert, "A nonlinear least-squares approach for estimation of the induction motor parameters", in Proceedings of the IEEE Conference on Decision and Control, December 2004, to appear. 